\title{
ECAM Control System Based on Auto-tuning PID Velocity Controller with Disturbance Observer and Velocity Compensator
}

\author{
Quang-Vinh Tran", Won-Ho Kim**, Jin-Ho Shin ${ }^{* *}$ and Woon-Bo Baek ${ }^{* *}$ \\ *Department of Intelligent System Engineering, Dong-Eui University \\ ** Department of Mechatronics Engineering, Dong-Eui University
}

\begin{abstract}
This paper proposed an ECAM (Electronic cam) control system which has simple and general structure. The proposed cam controller adopted the linear and polynomial curve-fitting method to generates a smooth cam profile curve function. Smooth motion trajectory of master actuator guarantees the good performance of slave motion and has an important effect on the interpolation quality of ECAM. The auto-tuning PID velocity controller was applied to overcome the uncertainties in ECAM, and the gains of the controller are updated continuously to ensure the consistency of system performance under varying working conditions. The robustness of system against the varying load torque disturbances and noises is guaranteed by using the load torque disturbance observer to suppress the disturbance on master actuator. The velocity compensator was applied to compensate the degradation of performance of slave motion caused from the varying driving speed of master motion. The stability and validity of the proposed ECAM control system was verified by simulation results.
\end{abstract}

Key Words : ECAM, Disturbance observer, Auto-tuning PID, Velocity compensation, Linear curve-fitting, Polynomial curve-fitting.

\section{Introduction}

ECAM (Electronic cam) is typically used to perform coordinated control. ECAM is a technique used to perform nonlinear motion electronically similar to that achieved with mechanical cams. ECAM control is a well-known masterslaves system in which one or more slave axes are driven to follow a predetermined trajectory as a function of a master axis position. It involves velocity tracking control of the master motor and trajectory generation of the slave motor. Nowadays ECAM which substitutes for mechanical cam is used in such applications as moving web handling, robotic material handling, and nonlinear machining. It has the advantage of software flexibility to quickly change from one cam profile to another, and it eliminates problems associated with the mechanical linkage, such as backlash, friction, engagement de-bouncing and structural resonance.

In order to generate cam profile, C. S. Liao, S. L. Jeng and W. H. Chieng [1], and Y. H. Chang et al.[4] proposed the linear and polynomial curve-fitting interpolation in which the estimated slave position is calculated from the predicted next position of the master. To guarantee the robust performance of cam following process, [1] referred to the electronic cam motion generation with special reference to constrained velocity, acceleration and jerk. In this paper, a load torque disturbance observer was used to suppress the disturbance on master actuator. While H. S. Yan, M. C. Tsai and M. H. Hsu

Manuscript received Nov. 27, 2009; revised Jan. 28, 2010

Accepted Feb. 12, 2010.

Corresponding Author : Won-Ho Kim(kwh@deu.ac.kr)

This work was supported by 2009 Dong-eui University Grant (grant number 2009AA166).
[2] claimed that the velocity and acceleration profiles were required to be continuous and to have small peak value and the jerk curve should be finite to minimize the vibrations caused by inertia forces, so they proposed the method to design the motion trajectory for reducing the peak values of motion characteristics. In Wang and Tsao [3], the repetitive controller was constructed from angle domain in which time-invariant dynamics of slave becomes time-varying. In this work, the model reference control was used to compensate for linear time- invariant model. Y. H. Chang et. al. [4] specified a novel master switching method that can help to avoid losing tracking precision when the load torque conditions of master and slave actuators are magnificently different or the actuators reach the limit of performance. This idea is specific to systems where the roles of master and slave motion are equivalent, but in most cases of cam follower application, it is impossible to exchange the roles between master and slave motion. Kim and Tsao [5] developed an ECAM motion generation on an electro-hydraulic servo actuators system including a repetitive controller which works as a Finite Impulse Response Filter and a feed-forward controller implements the zero phase error tracking as a supplement for feedback controller. However, hydraulic actuators have different dynamic characteristics relative to electric servo motors.

[1] did not consider the disturbance suppression and uncertainty on slave motion. [2] proposed the method to generate speed trajectory, but actuator dynamics, disturbance and uncertainty were not considered. [3] includes generally complex algorithm by designing the control system from the angle domain and using the two control terms : model reference control and repetitive controller to archive asymptotic output tracking and compensate for linear time-invariant model. [4] is only applicable for specific system that master and slave can 
exchange the roles. [5] is also specific for electro-hydraulic actuator, and has complex structure by using the two controllers: repetitive controller and feed-forward controller.

In this paper, we propose an ECAM control system based on auto-tuning PID velocity controller with load torque disturbance observer and velocity compensator. The most important characteristic of the proposed ECAM system is that it has a simple and general structure to implement system. i.e., the proposed system is based on PID controller that is easy to adjust gains. Moreover, we considered the disturbance suppression on slave motion as well as master motion. And we adopted the auto-tuning PID velocity controller to overcome the uncertainties. .

\section{Controller Scheme}

\subsection{The proposed Electronic CAM controller scheme}

The controller scheme is described in Fig. 1 in which the controller inputs are the reference feedback from master actuator and internal encoder feedback of slave actuator, and the output is the command voltage which drives slave motion.

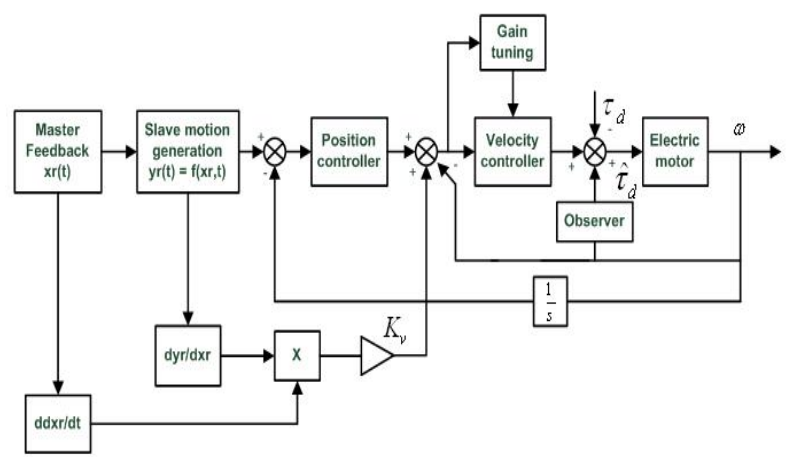

Fig. 1 The proposed ECAM control system.

Consider the dynamics model of an $\mathrm{AC}$ servo motor given as

$$
J \dot{\omega}+B \omega+\tau_{d}=\tau_{e}
$$

where $\tau_{e}$ is the electric torque control input, $\omega$ is the angular velocity of the rotor, $J$ and $B$ are the moment of inertia and damping coefficient of motor respectively, and $\tau_{d}$ can be considered as the load torque disturbance on the rotating shaft.

During ECAM operating process, the exact values of $J, B$, and $\tau_{d}$ are unknown, and $\tau_{d}$ is supposed to be bounded.

\subsection{ECAM profile generation}

The methods of ECAM profile generation are based on the prediction of the next master position:

$$
\hat{x}_{k+1}=x_{k}+\omega_{k} \Delta t
$$

where $x_{k}$ and $\omega_{k}$ are the current position and velocity of master motion, $\Delta t$ is the sampling time.

From the predicted next position of the master actuator, next reference position of the slave can be estimated by linear curvefitting or polynomial curve-fitting interpolation.

\subsubsection{Linear curve-fitting interpolation}

The piecewise linear curve-fitting method is developed to generate the piecewise cam trajectory as shown in Fig. 2.

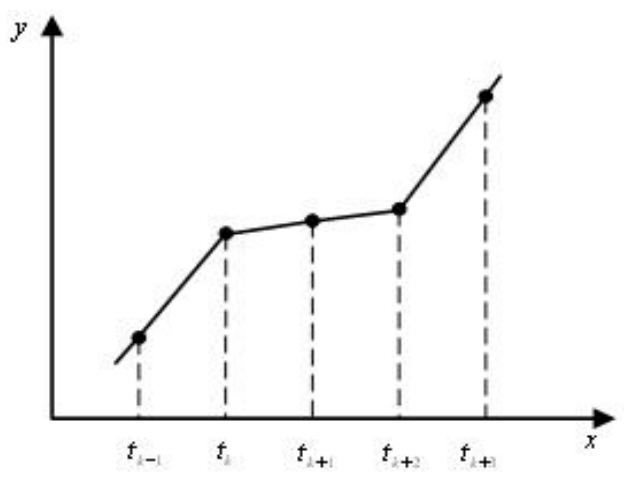

Fig. 2 Piecewise linear curve-fitting interpolation.

The predicted position of the slave can be determined as

$$
\hat{y}_{k+1}=\frac{\hat{x}_{k+1}\left(y_{i+1}-y_{i}\right)}{x_{i+1}-x_{i}}
$$

$k=0, \ldots, n-1$, with $n$ is the number of sampling points between $x_{i}$ and $x_{i+1}$.

\subsubsection{Polynomial curve-fitting interpolation}

The Lagrange $\mathrm{n}^{\text {th }}$ order polynomial formula is applied to build a continuous curve that fits the number of known discrete points (from ECAM data table). The polynomial interpolating method generates a smooth trajectory through the set of coordinates $\left(x_{i}, y_{i}\right)$ of master-slave as shown in Fig. 3.

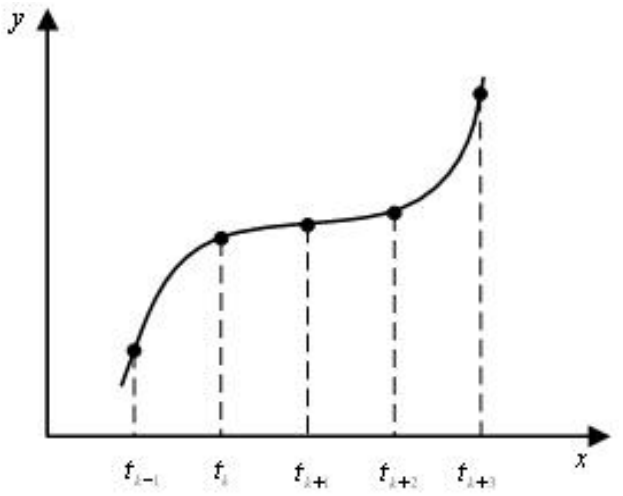

Fig. 3 Polynomial curve-fitting interpolation.

The $\mathrm{n}^{\text {th }}$ order Lagrange polynomial is expressed as

$$
f_{L}\left(x_{k}\right)=\sum_{i=0}^{n} L_{i}\left(x_{k}\right) y_{i},
$$


Where

$$
L_{i}(x)=\prod_{j=0, j \neq i}^{n}\left(\frac{x-x_{j}}{x_{i}-x_{j}}\right)
$$

The next ideal position of the slave motion is estimated as:

$$
\hat{y}_{k+1}=f_{L}\left(\hat{x}_{k+1}\right)=\sum_{i=0}^{n} L_{i}\left(\hat{x}_{k+1}\right) y_{i}
$$

\subsection{The velocity compensator}

During ECAM process, feedback position from master motion may vary due to the inconstant velocity of master actuator. The varying driving speed of master motion causes the error in next master position estimations that depicts in Fig. 4.

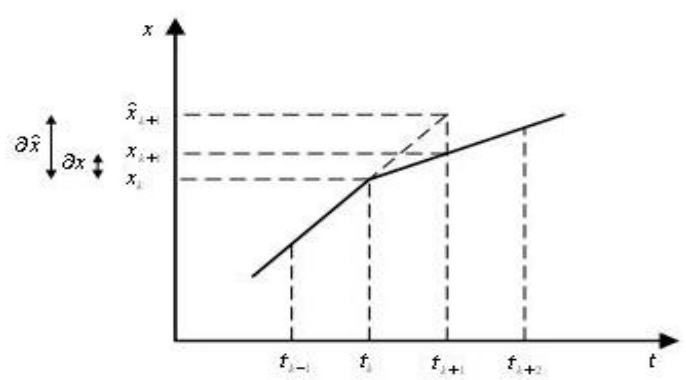

Fig. 4 Position estimation error of master motion

At the time $k+1$, the estimated position of the master drive is $\hat{x}_{k+1}=x_{k}+\omega_{k} \Delta t$, and the real one is $x_{k+1}$. If the velocity of the master is not constant, $\hat{x}_{k+1} \neq x_{k+1}$ and the velocity compensating value is calculated as:

$$
\omega_{\text {comp }}=\frac{(\delta x-\delta \hat{x}) \frac{\delta \hat{y}}{\delta \hat{x}}}{\delta t}
$$

The output of velocity controller is obtained by:

$$
u_{c}=f\left(e_{\omega}+\omega_{\text {comp }}\right)
$$

where $e_{\omega}=\omega_{c}-\omega$ is the angular velocity error and function $f$ is performed by the PID controller.

\subsection{Disturbance observation and suppression}

Disturbances on the slave motion may directly affect the performance of cam following routine. Therefore disturbances must be suppressed.

From the dynamics equation of induction AC servo motor (1), we can obtain $\tau_{d}=\tau_{e}-J \dot{\omega}-B \omega$.

Accordingly, the load torque can be estimated as

$$
\hat{\tau}_{d}=\tau_{e}-\hat{J} \dot{\omega}-\hat{B} \omega
$$

where $\hat{J}$ and $\hat{B}$ are the estimated moment of inertia and damping coefficient, and $\hat{\tau}_{d}$ is the estimated load torque disturbance.
The equation (8) for the estimated load torque disturbance is very numerically sensitive to random measurement noises because it yields high gains in the high-frequency field [1]. So a first-order low-pass filter is supplemented to estimate the load torque disturbances with the consideration of random noises as presented in Fig. 5.

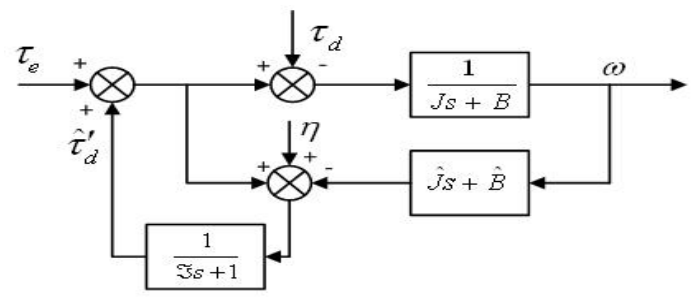

Fig. 5 The estimated load torque disturbance.

From Fig. 5, the final estimated load torque disturbance is determined as

$$
\hat{\tau}_{d}^{\prime}=\frac{1}{1+\mathfrak{I}_{S}}\left(\hat{\tau}_{d}+\eta\right)
$$

The estimation of the load torque disturbance is time delaying so that a correction factor $\eta$ is supplemented to compensate the measurement error caused by this timing mismatching. The disturbance compensating corrected value is determined depends on the state error of system:

$$
\eta=K_{e} e_{y}
$$

where $e_{y}=\hat{y}_{k}-y_{k}$ is the state error of system.

\section{Auto-tuning PID Controller}

For the purpose of developing a simple and effective controller scheme, the traditional PID controller is chosen for velocity controller of system. In cases that the parameters of actuator and working conditions are exactly known, a conventional PID controller can produce good performance of system. However, in most of real ECAM processes, the working conditions are inclined to be uncertain or unknown. So an auto-tuning PID controller is proposed to guarantee robust system performance against uncertainties.

The standard structure of a PID controller can be described in non-interacting form:

$$
G(s)=K_{C}\left(1+\frac{1}{T_{i} s}+T_{d} s\right)
$$

where $K_{C}$ is controller gain, $T_{i}$ is integral time, $T_{d}$ is derivative time, and $G(s)$ is the controller transfer function.

\subsection{Initialization}

There are many well known practical tuning methods which help to tune the gains of PID controller [10]. Some popular 
methods are introduced in Table. 1.

In Table $1, \mathrm{Ku}$ is the ultimate gain, at which the output of control loop starts to oscillate, and $\mathrm{Pu}$ is the oscillation period.

Each method has advantages and disadvantages and produce different results for different planning targets. In the simulation of this paper, the PID gains are pre-tuned by referring to standard Ziegler-Nichols tuning method combined with manual gain refining.

Table 1. PID gains tuning methods.

\begin{tabular}{|c|c|c|c|c|}
\hline Methods & $\begin{array}{c}\text { Controller } \\
\text { type }\end{array}$ & $K_{C}$ & $T_{i}$ & $T_{d}$ \\
\hline \multirow{2}{*}{$\begin{array}{c}\text { Ziegler- } \\
\text { Nichols }\end{array}$} & $\mathrm{P}$ & $\mathrm{Ku} / 2$ & -- & -- \\
\cline { 2 - 5 } & $\mathrm{PI}$ & $\mathrm{Ku} / 2.2$ & $\mathrm{Pu} / 1.2$ & -- \\
\cline { 2 - 5 } & $\mathrm{PID}$ & $\mathrm{Ku} / 1.7$ & $\mathrm{Pu} / 2$ & $\mathrm{Pu} / 8$ \\
\hline \multirow{2}{*}{$\begin{array}{c}\text { Tyreus- } \\
\text { Luyben }\end{array}$} & $\mathrm{PI}$ & $\mathrm{Ku} / 3.22$ & $\mathrm{Pu} / 0.45$ & -- \\
\hline \multirow{2}{*}{$\begin{array}{c}\text { Ciancone- } \\
\text { Marlin }\end{array}$} & $\mathrm{PID}$ & $\mathrm{Ku} / 2.2$ & $\mathrm{Pu} / 0.45$ & $\mathrm{Pu} / 6.3$ \\
\cline { 2 - 5 } & $\mathrm{PI}$ & $\mathrm{Ku} / 3.3$ & $\mathrm{Pu} / 4$ & -- \\
\hline
\end{tabular}

\subsection{Auto-tuning PID controller}

The auto-tuning PID controller is adopted to overcome the uncertainties in ECAM operating process, especially it is needed to suppress the variations of the slave estimated position value in the interpolating routine.

The update law of PID gains is proposed:

$$
\dot{K}_{c}(t)=K_{\delta} \frac{\delta^{2} \hat{y}}{\delta t^{2}}
$$

where $K_{\delta}$ is a positive constant, and $\delta \hat{y}=\hat{y}_{k}-y_{k-1}$ is the tracking position command error.

\section{Simulation Results}

A simulation program was made in Microsoft Visual $\mathrm{C}++$ 6.0 to validate the stability of the proposed controller. The working time of the simulated ECAM routine is 10 seconds with the sampling time is 0.01 second.

The parameters of slave actuator are determined as $B=0.0054 \mathrm{Nms} / \mathrm{rad}, \quad J=0.00478 \mathrm{kgm}^{2}$.

The master velocity is considered with variations:

$-t=0, x_{r}=0(\mathrm{rad})$,

$-t=3 s, x_{r}=1200(\mathrm{rad})$,

$-t=7 \mathrm{~s}, x_{r}=2000(\mathrm{rad})$,

$-t=10 \mathrm{~s}, x_{r}=3500(\mathrm{rad})$.

ECAM profile is defined in Table. 2:

The load torque disturbance is generated as $\tau_{d}=50 \sin (2 \pi t)+\operatorname{rand}(50)$ for the combination of periodic disturbance and random noises. The estimated parameters for the disturbance observer are chosen as
$\hat{B}=0.0045 \mathrm{Nms} / \mathrm{rad}, \quad \hat{J}=0.0038 \mathrm{kgm}^{2}$, and the bandwid -th of the first-order low-pass filter is set as $\mathfrak{I}=0.01 \mathrm{~s}$.

The controller gains are set to

$K_{P}=0.15, K_{I}=0.25, K_{D}=0.0015$,

$K_{\delta}=\left[\begin{array}{lll}0.012 & 0.001 & 0.0001\end{array}\right], K_{V}=0.5$. The correction gain of the disturbance observer is set as $K_{e}=0.54$.

Table 2. ECAM profile data.

\begin{tabular}{|c|c|c|}
\hline Point & Master position (rad) & Slave position (rad) \\
\hline 1 & 500 & 500 \\
\hline 2 & 1000 & 2000 \\
\hline 3 & 1500 & 3000 \\
\hline 4 & 2000 & 3000 \\
\hline 5 & 2500 & 2700 \\
\hline 6 & 3000 & 2900 \\
\hline 7 & 3500 & 3200 \\
\hline
\end{tabular}

Table 3 shows the different results of system using conventional PID controller and the proposed auto-tuning PID controller in the same operating condition. Table 4 and Table 5 present the simulation results using linear curve-fitting and polynomial curve-fitting interpolation. Fig. 6 describes the interpolation tracking position of two interpolation methods. In Fig. 6 (a) and (b), the left figures present the tracking position of master and slave motion according to operation time, and the right figures are the actual tracking position in the reciprocal master-slave coordination under the effect of disturbances and uncertainties. Fig. 7 and Fig. 8 depict the system performances in cases of linear and polynomial interpolation with and without load torque disturbance suppression. It can be seen from figures and results that the system performance was improved significantly when apply the proposed controller with auto-tuning PID velocity controller and disturbance observer.
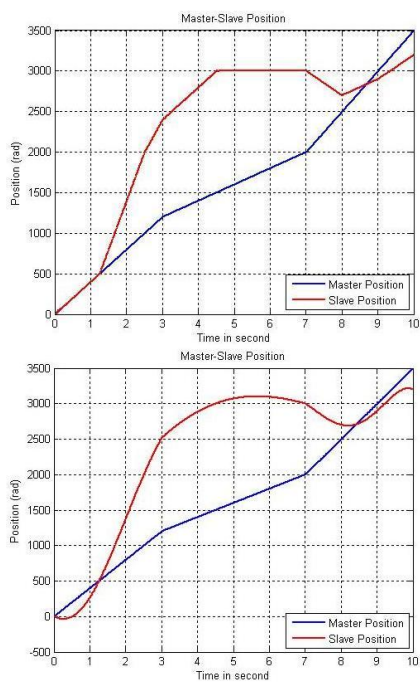

(a)
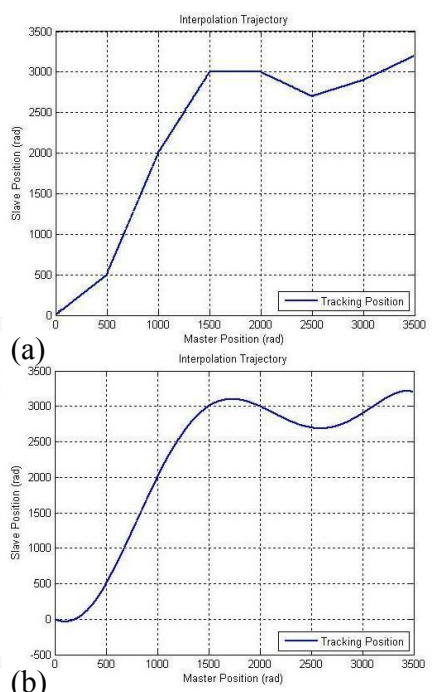

Fig. 6 Tracking position of (a) linear curve-fitting and (b) polynomial curve-fitting interpolation. 


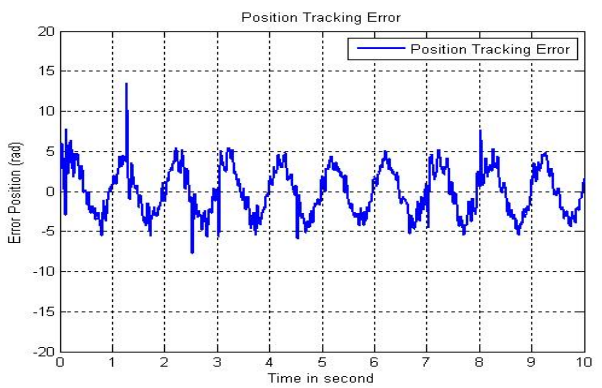

(a) without disturbance observer

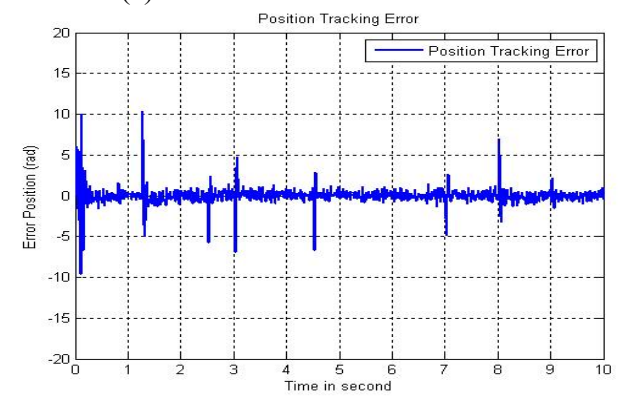

(b) with disturbance observer.

Fig. 7 Position tracking error using linear interpolation

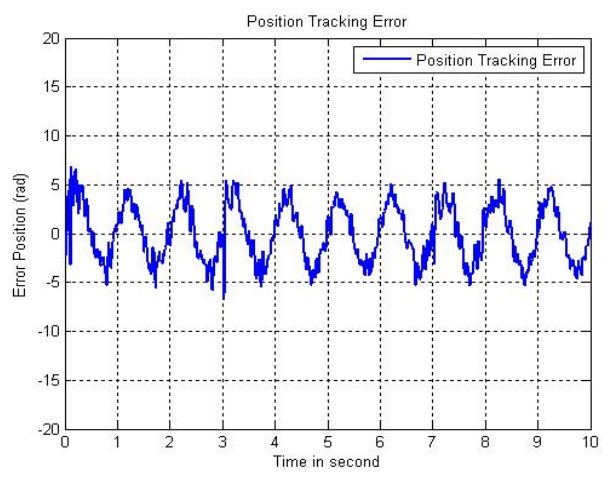

(a) without disturbance observer,

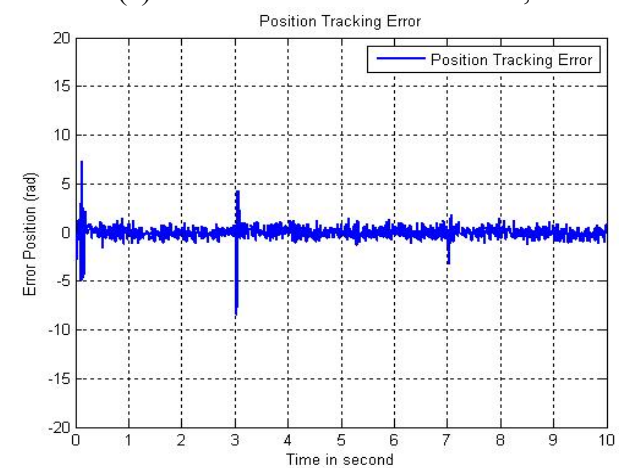

(b) with disturbance observer.

Fig. 8 Position tracking error using polynomial interpolation

Table 3. Simulation results in consideration of auto-tuning PID.

\begin{tabular}{|c|c|c|}
\hline Controller & $\begin{array}{c}\text { Position tracking } \\
\text { RMS error(rad) }\end{array}$ & $\begin{array}{c}\text { Position tracking } \\
\text { mean error(rad) }\end{array}$ \\
\hline Conventional PID & 1.190 & 0.641 \\
\hline Auto-tuning PID & 1.162 & 0.635 \\
\hline
\end{tabular}

Table 4. Simulation results of linear curve-fitting interpolation

\begin{tabular}{|c|c|c|}
\hline Controller & $\begin{array}{c}\text { Position tracking } \\
\text { RMS error(rad) }\end{array}$ & $\begin{array}{c}\text { Position tracking } \\
\text { mean error(rad) }\end{array}$ \\
\hline $\begin{array}{c}\text { Auto-tuning PID } \\
\text { without DO }\end{array}$ & 2.984 & 2.557 \\
\hline $\begin{array}{c}\text { Auto-tuning PID } \\
\text { with DO }\end{array}$ & 1.118 & 0.601 \\
\hline
\end{tabular}

Table 5. Simulation results of polynomial curve-fitting interpolation

\begin{tabular}{|c|c|c|}
\hline Controller & $\begin{array}{c}\text { Position tracking } \\
\text { RMS error(rad) }\end{array}$ & $\begin{array}{c}\text { Position tracking } \\
\text { mean error(rad) }\end{array}$ \\
\hline $\begin{array}{c}\text { Auto-tuning PID } \\
\text { without DO }\end{array}$ & 2.852 & 2.509 \\
\hline $\begin{array}{c}\text { Auto-tuning PID } \\
\text { with DO }\end{array}$ & 0.797 & 0.485 \\
\hline
\end{tabular}

It can be seen from the simulation results that the state errors caused by the piece-wise linear curve-fitting interpolation can be suppressed by using the polynomial curve-fitting interpolation while the estimated errors caused by the sudden changes of master speed remain the effects on slave motion. It can deduce that if the master motion speed varying is gradual so the state error of slave motion will be improved significantly. Fig. 9 and Table. 6 show the performances of system when the master motion trajectory profile is smoothened.

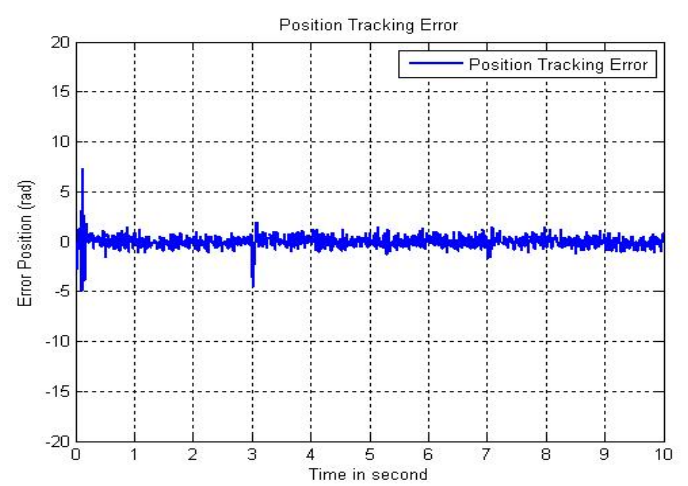

Fig. 9 Position tracking error using polynomial interpolation and master position trajectory is smoothened.

Table 6. Simulation results in consideration of master position trajectory.

\begin{tabular}{|c|c|c|}
\hline Controller & $\begin{array}{c}\text { Position tracking } \\
\text { RMS error(rad) }\end{array}$ & $\begin{array}{c}\text { Position tracking } \\
\text { mean error(rad) }\end{array}$ \\
\hline $\begin{array}{c}\text { Rough master } \\
\text { position trajectory }\end{array}$ & 0.797 & 0.485 \\
\hline $\begin{array}{c}\text { Smooth master } \\
\text { position trajectory }\end{array}$ & 0.689 & 0.465 \\
\hline
\end{tabular}

\section{Conclusion}

We have proposed an effective electronic CAM control 
system based on auto-tuning PID velocity controller with load torque observer and velocity compensator mechanism. The controller is shown to be stable, feasible and effective with the simulation results. It can perform error converging control and suppress disturbance sources to generate an adequate ECAM profile interpolation. This work has the advantage of simple structure and calculation that can be easily implemented in realtime using general microprocessors. In the future work, practical implementation will be carried out to consolidate the stability and feasibility of the proposed ECAM control system.

\section{Reference}

[1] C. S. Liao, S. L. Jeng, W. H. Chieng, "Electronic cam motion generation with special reference to constrained velocity, acceleration, and jerk", ISA Transaction, vol. 43, pp. 427-443, 2004.

[2] H. S. Yan, M. C. Tsai, M. H. Hsu, "An experiment study of the effects of cam speeds on cam-follower systems", Mech. Mach Theory, vol. 31, no. 4, pp. 397-412, 1996.

[3] J. Wang, T. C. Tsao, "Repetitive control of linear time varying systems with application to electronic cam motion control ", Proceeding of the 2004 American Control Conference, Boston, Massachusetts, June 30-July 2, 2004.

[4] Y. H. Chang, W. H. Chieng, "A novel master switching method for electronic cam control with special reference to multi-axis coordinated trajectory following", Control Engineering Practice, vol. 14, pp. 107-120, 2006.

[5] D. H. Kim, T. C. Tsao, "Robust performance control of electrohydraulic actuators for electronic cam motion generation", IEEE Transaction on Control System Technology, vol. 8, no. 2, March 2000.

[6] K. Hong, K. H. Nam, "A load torque compensation scheme under the speed measurement delay", Transactions on Industrial Electronics, vol. 45, no. 2, April 1998.

[7] M. Mizuochi, T. Tsuji, K. Ohnishi, "Improvement of disturbance suppression based on disturbance observer", Proceeding of the 9th International Workshop on Advanced Motion Control (AMC'06), Istanbul, Turkey, March 2006.

[8] Y. P. Yang, C. F. Fang, "Adaptive speed control of AC servo induction motors with on-line load estimation", Proceeding of the American Control Conference, Baltimore, Maryland, June, 1994.

[9] Z. Iwai, I. Mizumoto, L. Liu, S. L. Shah, H. Jiang, “Adaptive stable PID controller with parallel feedforward compensator", Proceeding of the 9th International Conference on Control, Automation, Robotics and Vision (ICARCV), Grand Hyatt, Singapore, December 2006.

[10] W. Tan, J. Z. Liu, T. W. Tan, H. J. Marquez, "Comparison of some well-known PID tuning formulas", Computers and Chemical Engineering, vol. 30, pp. 1416-1423, 2006.

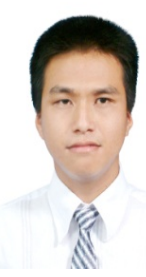

Quang-Vinh Tran received the B.S. degree in Department of Mechatronics Engineering from Ho Chi Minh City University of Technology, Vietnam in 2007. He is currently studying M.S. Course in Department of Intelligent System Engineering, Dong-Eui University, Busan, Korea. His research interests include motion control, robotics, embedded systems, and intelligent control theories.

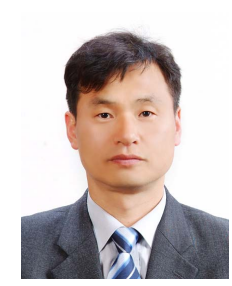

Won-Ho Kim received the B.S., M.S. and Ph.D. degree in School of Electronics Engineering from Kyungpook National University, Daegu, Korea, in 1985, 1988 and 1999, respectively. From 1988 to 1993, he was a researcher of Electronics and Telecommunications Research Institute (ETRI). He is currently a professor in the Department of Mechatronics Engineering, Dong-Eui University, Busan, Korea. His research interests include robotics and embedded control systems.

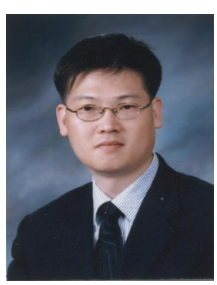

Jin-Ho Shin received the B.S. degree in Department of Electronic Engineering from Hanyang University, Seoul, Korea, in 1991, M.S. and Ph.D. degrees in Department of Electrical Engineering from KAIST, Daejeon, Korea, in 1993 and 1999, respectively. From 2000 to 2002, he was a JSPS postdoctoral research fellow in Department of MechanoInformatics, Graduate school of Engineering from University of Tokyo, Japan. He is currently an associate professor in the Department of Mechatonics Engineering from Dong-Eui University, Busan, Korea. His research interests include robotics, intelligent control systems.

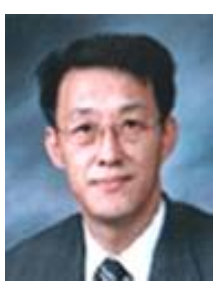

Woon-Bo Baek received the B.S., M.S. and Ph.D. degree in Department of Mechanical Engineering from Busan National University, Busan, Korea, in 1985, 1987 and 1993, respectively. From 1993 to 1999, he was a senior researcher of Machinery and Electronics Research Institutes, Samsung Heavy Industries Co. Ltd., Korea. From 2000 to 2002, he was a senior researcher of Research \& Development Center, Doosan Heavy Industries \& Construction Co. Ltd., Korea. He is currently an associate professor in the Department of Mechatonics Engineering from Dong-Eui University, Busan, Korea. His research interests include intelligent robot, robust nonlinear control and intelligent vehicle control. 